\title{
A CFD-FEM Approach to Study Wing Aerodynamics under Deformation
}

\author{
Luca Andreassi, Vincenzo Mulone, Pier Paolo Valentini, Leonardo Vita \\ University of Rome Tor Vergata
}

Copyright $\odot 2004$ Society of Automotive Engineers, Inc.

\begin{abstract}
Computational Fluid Dynamics (CFD) surely has played a fundamental role in the design of the bodies and shapes of both commercial and racing vehicles in the last decades. This circumstance was mainly due to the connected substantial improvement in the design timings and to the possibility of producing numerous flow field and surface data that are difficult to obtain from a physical experimental method. Such a local analysis leads to a further understanding of the interactions of components with the overall aerodynamics.
\end{abstract}

The development of wing performances, with respect to racing vehicles, has to deal with very short times but also with a very detailed description of the physics occurring. Starting from these constraints, a coupling procedure was developed by the joining of a CFD code with a Finite Element Method (FEM) structural code to better evaluate the aerodynamic performance of the wing deformed under the fluid dynamic loads. Combined with experimental data, these insights allow a better understanding of the involved flow mechanisms

The codes coupling has been managed as it follows: the fluid dynamic pressure loads have been passed to the structural solver. The output, in terms of airfoil deformation, has been re-imported into the CFD solver in order to compute the updated fluid dynamic field. These steps have to be repeated till the reaching of the convergence of the fluid dynamic and deformation fields. The final result consists of the fluid dynamic field around the deformed configuration of the wing.

The described procedure has been applied to a standard racing vehicle front wing, which was tested under different operating conditions in terms of vehicle speed and angle of attack. Results obtained are encouraging and suggest to further investigate the possibilities offered by this procedure.

\section{INTRODUCTION}

The efficiency of the aerodynamic package is a very important issue to obtain good performances from a racing car. The efforts of the aerodynamic engineers are mainly concentrated on the performance of a chassis in terms of two expressive forces:

- Downforce (negative lift), which is useful to force the car to the ground, and then to maintain high speed along the curves $[1,6,8,15]$;

- Drag, which is basically unwanted, since it slows the car.

The capability of an aerodynamic engineer is strictly related to the achievement of a good compromise between lift and drag, or, in other words, to obtain the desired lift with the lowest drag as possible.

The efficiency of a wing in general depends on the following items:

- Aspect ratio (length-width ratio). The amount of downforce produced by a wing is determined by its size. The larger the wing the greater is the downforce. The higher is the aspect ratio the more efficient is the wing because of less air resistance created by the vortex at the wing tips;

- Angle of attack. The greater the angle of attack, the greater is the downforce until stall, the greater is the unwanted drag;

- Drag. Increasing downforce, a wing also increases unwanted drag. Drag increases with the angle of attack.

The design of the front wing of a racing car (Fig. 1) constitutes particularly an important step since it is the very first part of the car to meet the flow, and then it has to be designed not only to produce the downforce (about $25 \div 40 \%$ of the total downforce offered by the entire car), but also to guide in an efficient way the flow toward the body, the underbody, and the rear of the car. Moreover, the front wing affects noticeably the flow approaching to the rear of the car, so strongly determining also the performance of the rear wing. 


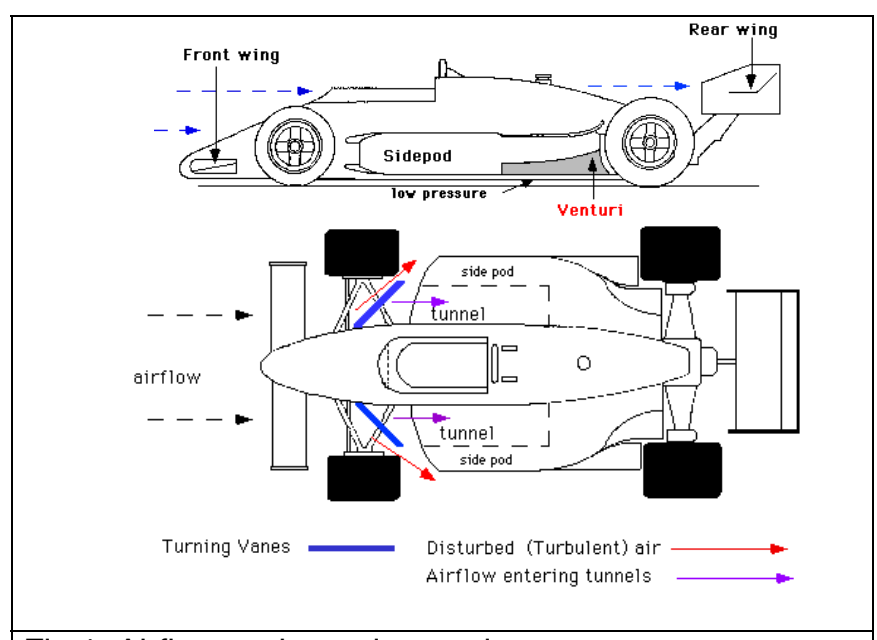

Fig 1: Airflows scheme in a racing car

Each front airfoil is made of one or more mainplanes of carbon fibre almost covering the entire width of the car, and generally has one full spanning flap; endplates constitute the boundaries of each mainplane. The performance of the front-wing changes radically with the closeness to the ground for the well-known "ground effect"; the distance between the wing and the ground is usually limited by specific competition rules.

Developments of the wing design usually concentrate on the wing profile and the flaps; moreover, some teams recently angled the leading edge to form a forwardfacing V-shape, in order to exploit the full potential of the wing: flow visualizations in fact showed that the suction power of the wing is so strong that air approaches to the leading edge with a different angle from the one presented by the undisturbed flow. Endplates design tends to change a lot: their primary function consists in separating the high pressure air (top of the wing) from the low pressure air (bottom of the wing); some teams also use "splitters" attached to the undersurface of the wing to assist the endplates.

Previous technical discussion surely makes clear the complexity of an efficient design of a racing car front wing and then the more expensive cost than a rear wing (about $\$ 21500$ a front wing; about $\$ 12500$ a rear one). CFD then would be an ideal tool for design, and it is already used and growing in importance because it allows reducing times and costs within such a complex scenario. In fact, firstly the performance of a number of possible configurations of the wing can be evaluated with the aid of a CFD solver; then the most efficient configurations undergo wind-tunnel [18] tests after which the optimal solution can be chosen.

According to the preceding analysis, the availability of more and more reliable CFD solvers and solution procedures constitutes surely a topic of interest $[16,17]$. In this paper, a numerical procedure will be presented: it has been developed by the coupling of a CFD and FEM solvers to evaluate the aerodynamic performances of a wing deformed under the fluid dynamic loads. The described procedure has been applied to a standard racing vehicle front wing, by varying the most important operating parameters such as speed and angle of attack.

\section{CFD-FEM COUPLING METHODOLOGY}

The definition of a correct, fast, accurate and easy to be implemented methodology is a crucial point $[11,17]$. The developed methodology is based on the integration between CFD model and FEM model. The codes used are the commercial software FLUENT for the fluid dynamic analysis and MSC-NASTRAN as structural solver: some details of the codes will be provided in the following sections. The proposed methodology can be summarized in the algorithm sketched in Figure 2. The final solution is found performing an iterative analysis as follows:

1. The wing original physical structure is analysed by means of the 3D CFD code to define the pressure field around the structure;

2. The pressure field is converted into nodal loads to apply to the finite element model;

3. A linear elastic analysis is performed in order to find the deformation of the entire wing under the nodal loads defined in the previous step;

4. The deformations evaluated in the previous step are processed by an algorithm program [5] in order to update the entire fluid dynamics volume mesh;

5. CFD analysis is again performed to define the updated pressure field around the deformed structure.

These operations are repeated until the convergence, i.e. the same deformation of the previous step within a predefined tolerance is obtained.

In order to perform the described integrated analysis, some main aspects have to be properly defined. First of all, a procedure which allows to exchange pressure and deformation fields between the solvers has to be defined. To this aim several subroutines have been linked to the main code, available on request. The most important of these translators are the algorithms sketched Figure 3 and Figure 4, which operate basically a linear interpolation [5] of the needed information on the wing surface, so allowing the use of different structural and fluid dynamic computational grids.

\section{BASICS OF THE CODES}

\section{FEM STRUCTURAL CODE}

The geometry of the wing has been splitted into several surfaces which have been defined using their bounding curves (non uniform B-splines) and a cubic interpolation. The entities have been exported in ACIS format [4] in 
order to preserve their topological information about the hierarchy and orientation.

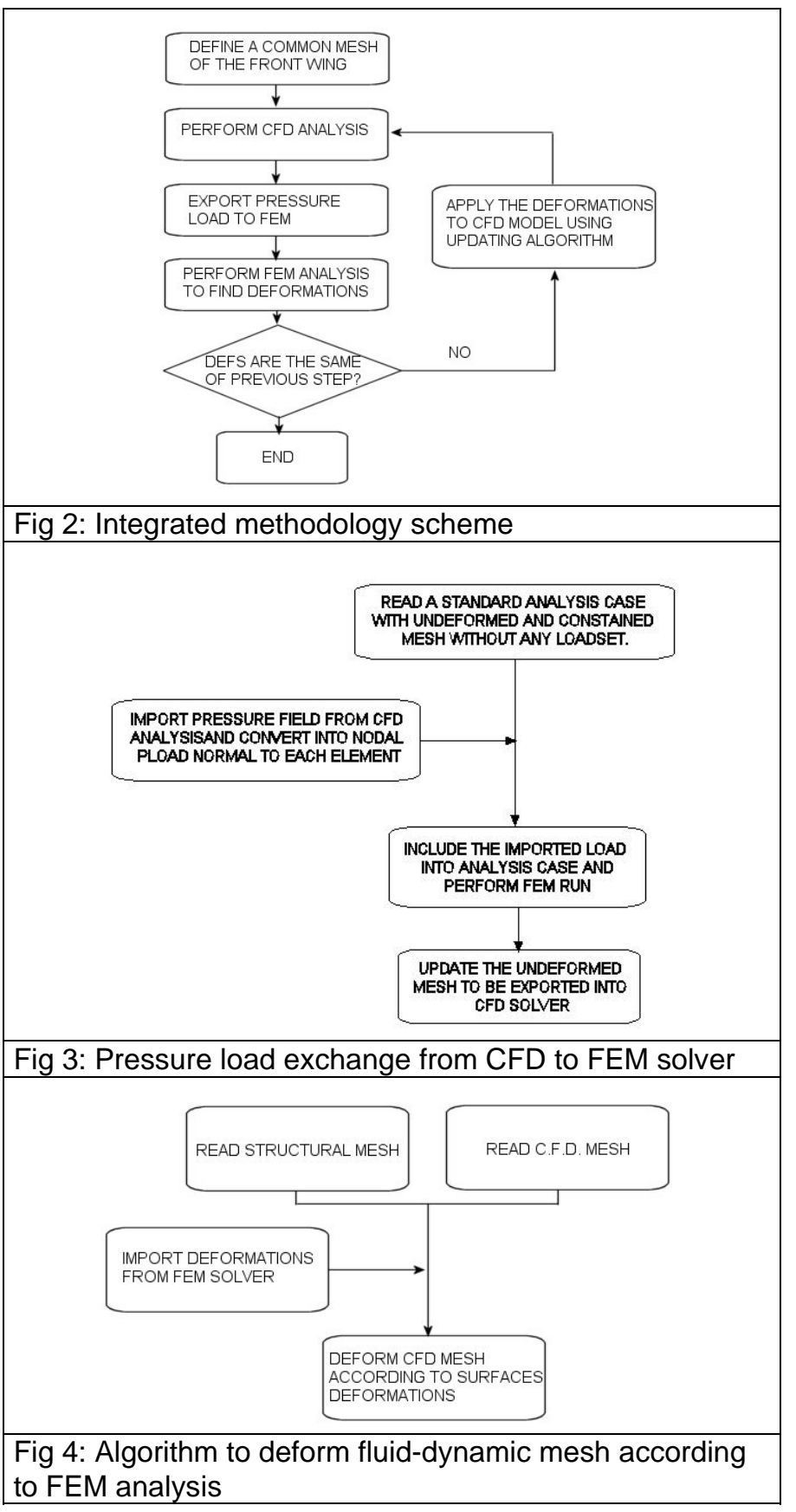

In order to study the deformation under pressure loads, the front wing has been modelled by means of finite element technique. The system has been simulated using triangular planar element with 5 d.o.f. each node [19]. Only an half of the entire wing has been meshed, because of the symmetry of the geometry, the loads and the constrains. The model has been constrained in the cutting plane to preserve the above mentioned conditions. Moreover, the node of the vertical anchor segment to the nose has been fixed to the ground. The material used is a generic CFRP with not oriented fiber, so that it could be modelled as an isotropic linear elastic material, characterised by the following properties:

- Young's modulus (E): 2.28E+11 Pa

- Shear modulus (G): 1.03E+10 Pa

- Poisson ratio (v): 0.27

- Mass density $(\rho): 1580 \mathrm{~kg} / \mathrm{m}^{3}$

An overall picture of the undeformed mesh is presented in Figures 5 and 6.
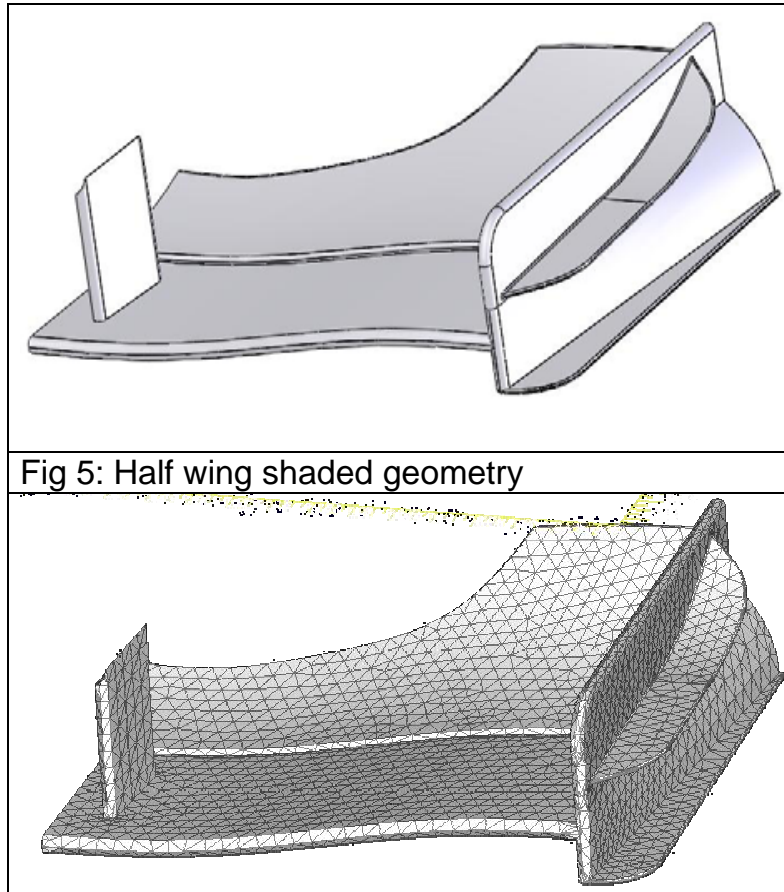

Fig 6: Half wing mesh

\section{FLUID DYNAMIC CODE}

Fluent CFD code [20] was used to simulate the fluid dynamics around the wing. Fluent solves the classical basic fluid-dynamics set of equations (mass, momentum and energy) omitted here for the sake of brevity. Since the speed of the vehicle is very high, surely it induces turbulent phenomena around the wing; then additional equations are required to simulate these not negligible effects. Two equations models are implemented into Fluent; in particular Standard, RNG and Realizable k- $\varepsilon$ models were used. All the cited models are linked to the fluid dynamics equations by means of the classical turbulent viscosity hypothesis, which is substantially the following:

$$
v_{t}=C_{\mu} \frac{k^{2}}{\varepsilon}
$$


The three models differ for the analytical formulation, and also for the definition of the turbulent viscosity (Standard: $\mathrm{C}_{\mu}=0.09$; RNG: $\mathrm{C}_{\mu}=0.0845$, totally different formulation for Low-Re fluxes; Realizable: $\left.C_{\mu}=C_{\mu}(x, t)\right)$. Inlet boundary conditions on turbulent quantities are defined on the basis of a percentage of the average kinetic energy $T_{\text {in }}=0.5 u_{i} u_{i}$ and of a characteristic length $L$ by means of the following hypothesis:

$$
\begin{gathered}
k_{i n}=f \times T_{i n} \\
\varepsilon_{i n}=\frac{C_{\mu}^{3 / 4} \times k_{i n}{ }^{3 / 2}}{0.07 L}
\end{gathered}
$$

(f assumes values in the range $0.01 \div 0.1$, depending on the inlet turbulence level)

The 3D computational mesh together with the appropriate boundary conditions were built with Gambit; the mesh is constituted by tetrahedral elements: this circumstance in fact allows to obtain a quite refined grid near the wing surface which surely constitutes the portion of the domain most interested by high variablegradients. Since turbulence plays a dominant role in the transport of mean momentum and other scalars, it has been necessary to be sure that turbulent quantities are properly resolved. For this reason an evaluation of the distance from the wall at the wall-adjacent cells has been done. This distance $y^{+}=\frac{\rho u_{T} y}{\mu}$ was checked to be between 30 and 60, values for which the log-law is retained valid. An external FORTRAN program was further implemented to rotate the keying angle (with respect to the ground) without any additional efforts on the mesh definition.

\section{$\underline{\text { CFD code validation }}$}

A basic CFD test was managed in order to prove the predictive capabilities of the code in a situation similar to the wing simulation. A 2D simulation of a cascade of compressor blades was chosen, since experimental data were available with respect to NACA 65 series [7].

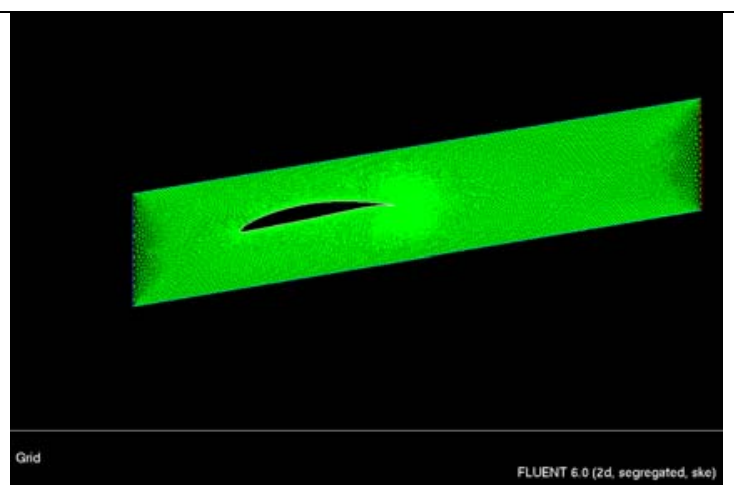

Fig. 7: NACA 65 2D computational mesh
The 2D mesh is constituted by triangle elements: a sketch of the grid is provided in Fig.7. The main fluid dynamic operating conditions of the tested case are displayed in Table 1; Fig.8 defines the flow angles.

\begin{tabular}{|l|l|}
\hline Blade & Naca $65-810$ \\
\hline Blade chord & $\mathrm{C}=13 \mathrm{~cm}$ \\
\hline Solidity & $\sigma=1.0$ \\
\hline Re & 300000 \\
\hline Inlet angle & $\beta_{1}=30^{\circ}$ \\
\hline Attack angle & $\alpha_{1}=9.7^{\circ}$ \\
\hline Inlet velocity & $\mathrm{V}=29 \mathrm{~m} / \mathrm{s}$ \\
\hline Inlet temperature & $300 \mathrm{~K}$ \\
\hline Outlet pressure & 101325 Pascal \\
\hline Inlet turb kin energy & $0.1 \mathrm{u}_{i} \mathrm{u}_{\mathrm{i}}$ \\
\hline Characteristic length & $13 \mathrm{~cm}$ \\
\hline
\end{tabular}

Tab. 1

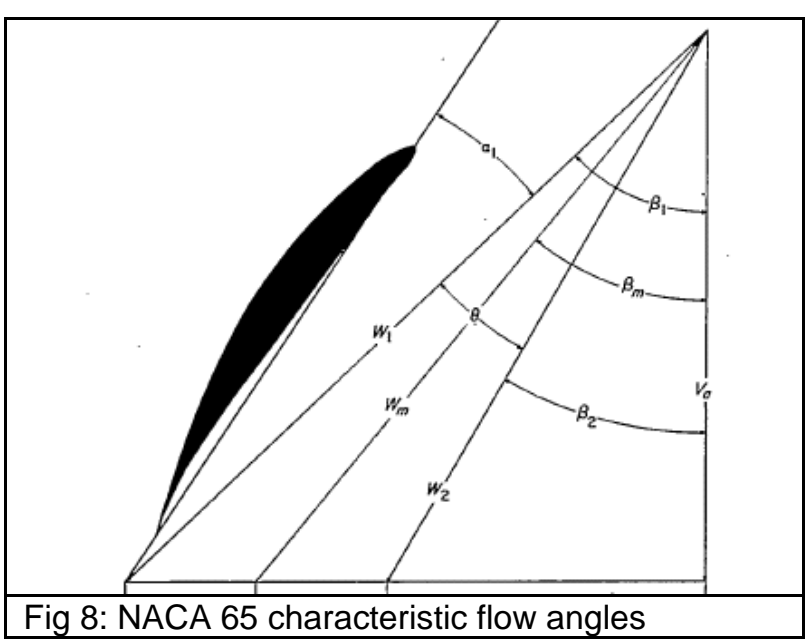

Results (Fig.9) are presented in terms of pressure coefficient $S=1-\frac{p-p_{0}}{0.5 \rho_{0} u_{0}^{2}} \quad$ distribution along the concave and convex surfaces of the blade. The comparison between experimental and numerical data was quite satisfactory. Moreover, little changes are observed for the trend of these quantities by varying turbulence model.

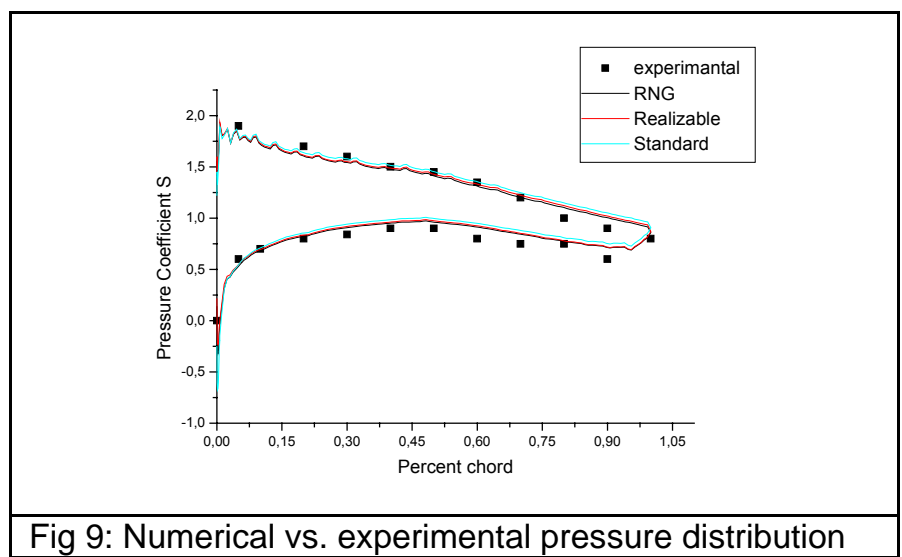




\section{ANALYSIS OF RESULTS}

The procedure previously exposed was applied to the study of a typical Formula 1 car front wing.

A front view of the wing is displayed in Fig.10: it exhibits a "spoon" shape, i.e. it is slightly lower next to the symmetry axis. According to Formula 1 regulations, and with the additional hypothesis that the car flat bottom lies at $15 \div 20 \mathrm{~mm}$ from the track, the distance between the lowest portion of the wing and the track was assumed equal to $11.7 \mathrm{~cm}$.

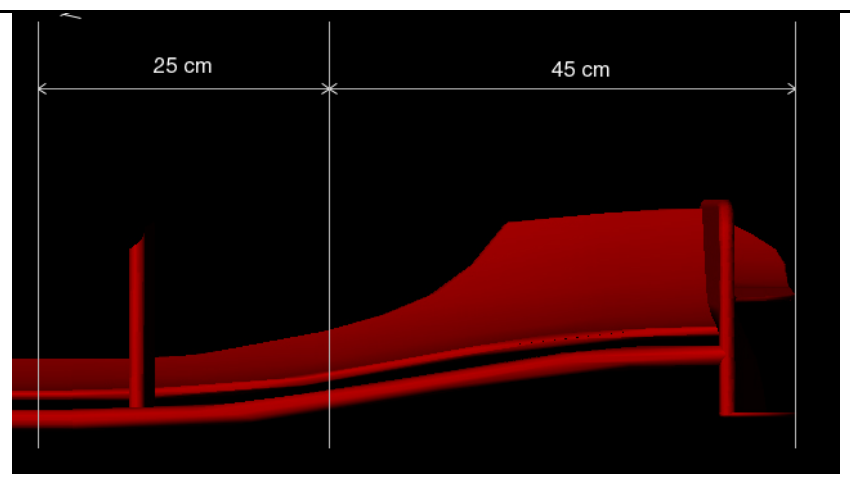

Fig 10: Wing front view

Main operating conditions are the same discussed previously but the turbulence intensity was fixed to $5 \%$ of inlet kinetic energy as the numerical simulation refers to a quiescent ambient atmosphere; RNG k- $\varepsilon$ model was basically chosen, even if a brief discussion on turbulence models can be found below.

\section{RIGID WING FLUID DYNAMIC ANALYSIS}

Pure fluid dynamics results are here displayed for the rigid geometry: the analysis was performed for speeds up to $300 \mathrm{~km} / \mathrm{h}$.

Lift and drag are reported in Fig. 11, while some sketches concerning pressure fields are reported in Figures 12 and 13

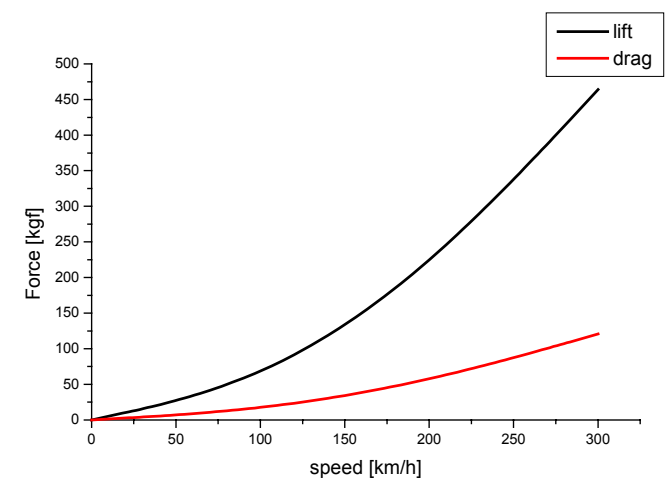

Fig 11: Lift and drag as function of the speed

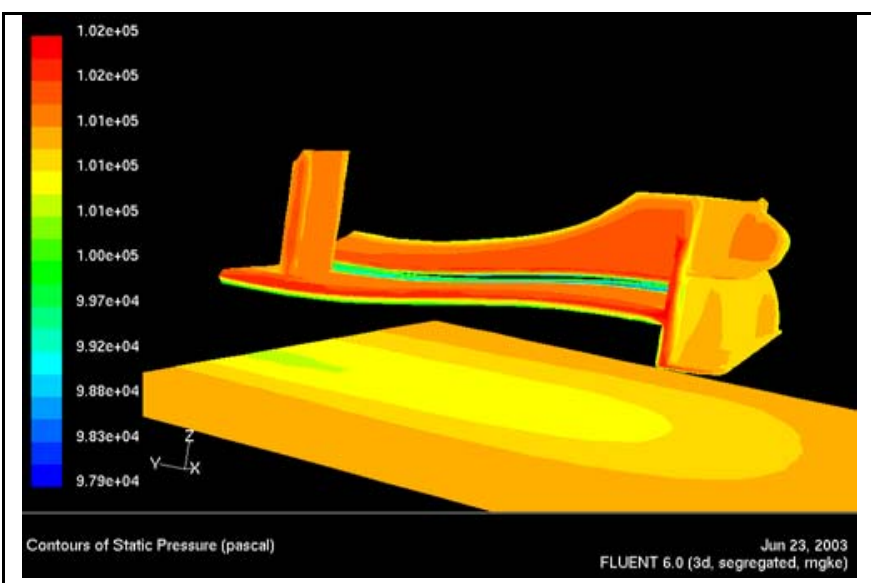

Fig 12: Static pressure field on the wing at $100 \mathrm{~km} / \mathrm{h}$

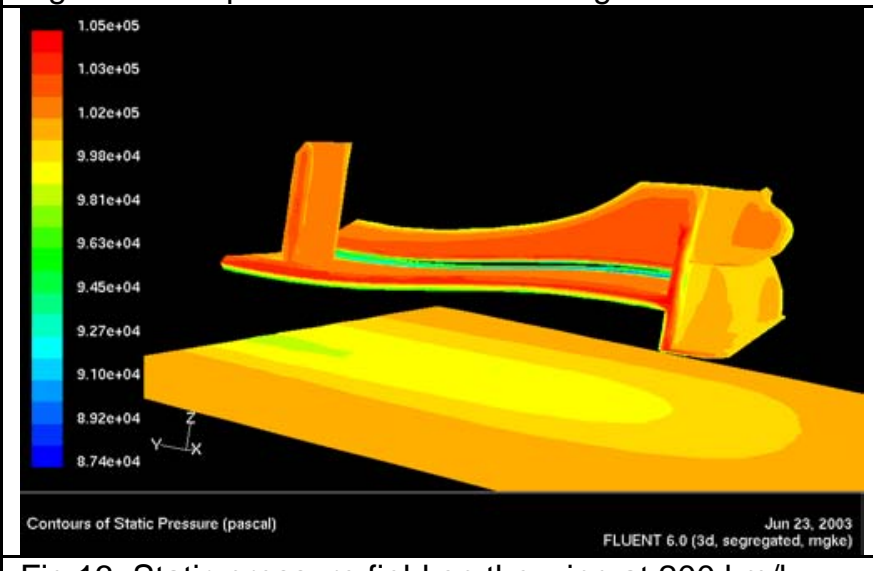

Fig 13: Static pressure field on the wing at $200 \mathrm{~km} / \mathrm{h}$

The analysis was further applied to the study of the performance by slightly varying the pitch angle of the wing of \pm 1 and \pm 2 degrees, so simulating the presence of possible installation errors. The results in terms of forces and percentages are displayed in Fig. 14 and Fig. 15 only for positive angle errors, for the sake of brevity.

Finally, the influence of the turbulence model was investigated: results in terms of lift and drag are presented for Standard, RNG and Realizable k- $\varepsilon$; the substantial equality of the trends and the values observed in Fig.16 justifies the choose of only one of the models: the RNG was chosen because it represents a compromise between accuracy and time-cost among the three proposed models.

\section{DEFORMED WING FLUID DYNAMIC ANALYSIS}

\section{FEM structural analysis}

The presented fluid dynamics analysis allowed the evaluation of the pressure field on the overall surface of the wing. These data were used to perform a stressstrain analysis of the wing; the details about the geometry, material and constraints have been discussed in the previous section. 


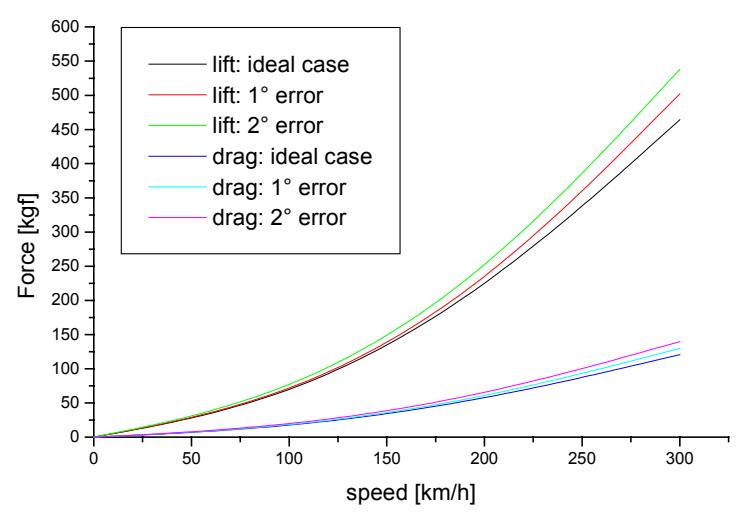

Fig 14: Lift and drag as function of the speed by varying pitch angle

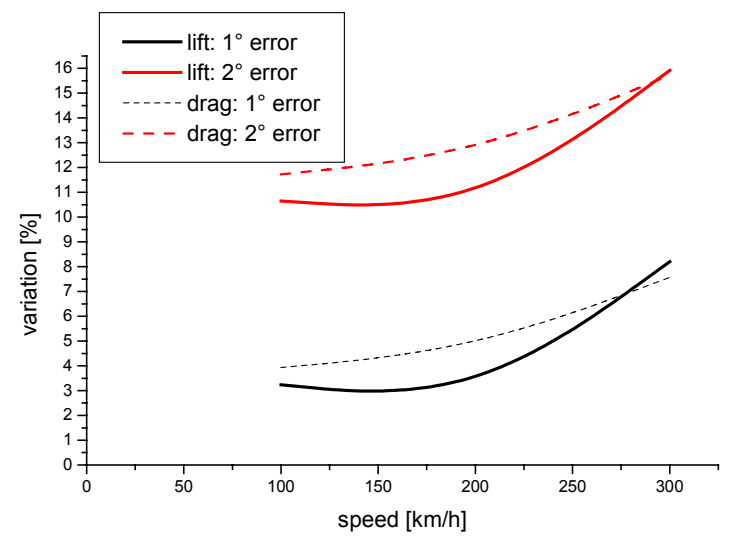

Fig 15: Percentage variation of lift and drag by varying the pitch angle

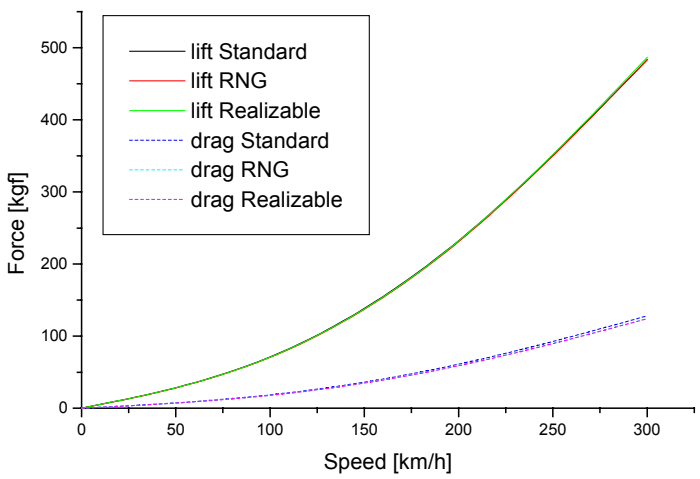

Fig 16: Lift and drag trends by varying turbulence models

In Figure 17 the Von Mises contour plot computed at $300 \mathrm{~km} / \mathrm{h}$ is depicted. The critical areas are the lower airfoil and the nose connection zone where elements shows higher stress values. In this case the deformation of the wing (shown in Figure 18 and 19) decreases the attack angle and explains the changing of dragdownforce values (Figure 11, 14, 15 and 16). The maximum nodal displacement is about $0.8 \mathrm{~cm}$.
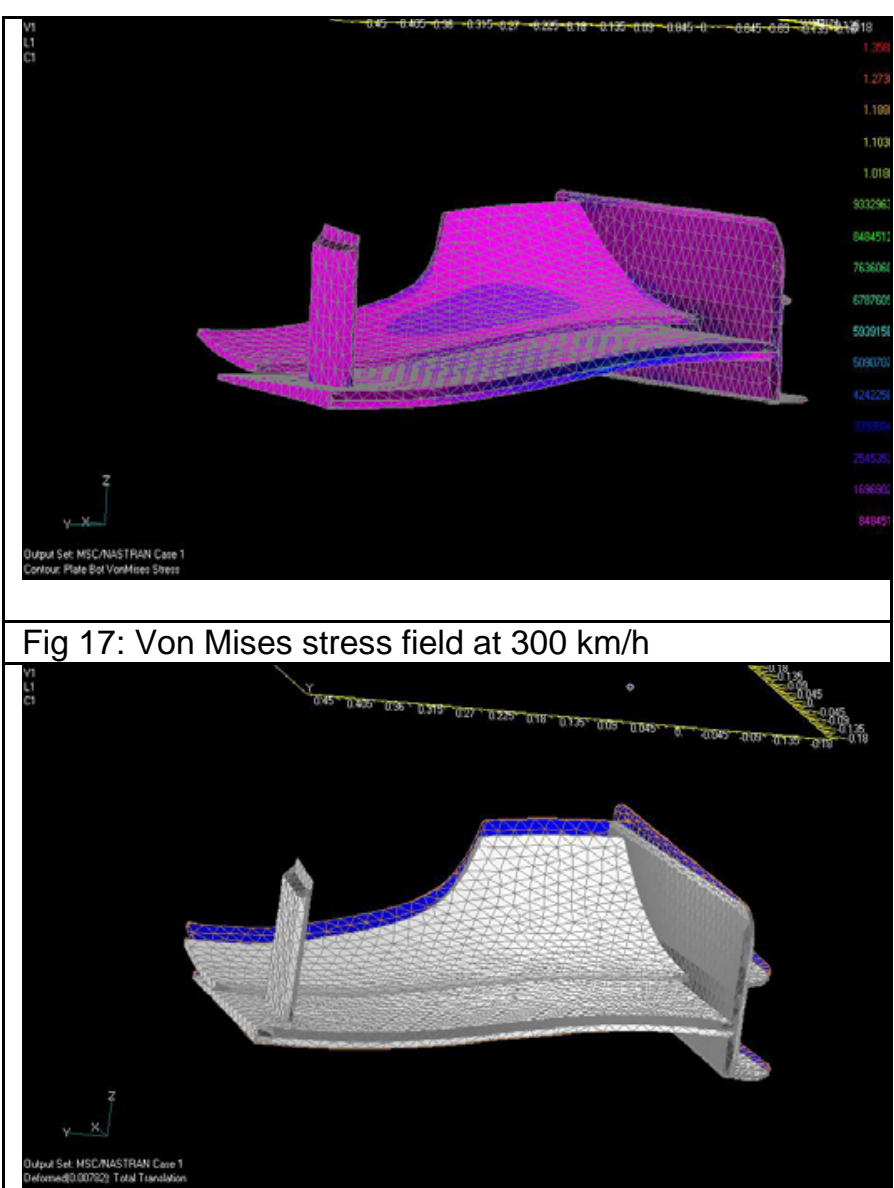

Fig 18: Overlapping of deformed (grey) and undeformed (blue) geometries: deformation is magnified by the $300 \%$

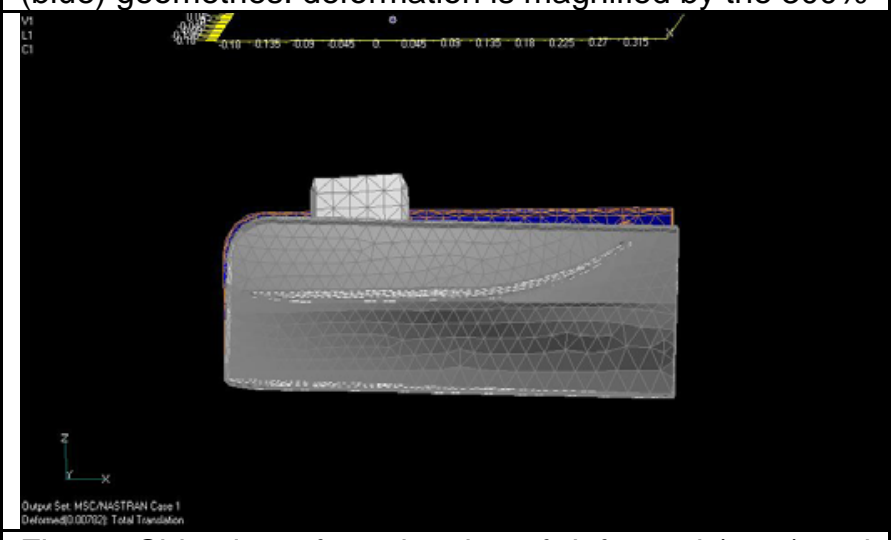

Fig 19: Side view of overlapping of deformed (grey) and undeformed (blue) geometries: strain is magnified by the $300 \%$

\section{Fluid dynamics analysis of the deformed wing}

Finally, the two codes were joint by following the procedure exposed in the previous section to evaluate the actual performance of the wing in its deformed configuration.

First of all the trend of the force vs. the number of iterations is provided in Figures 20 and 21. 


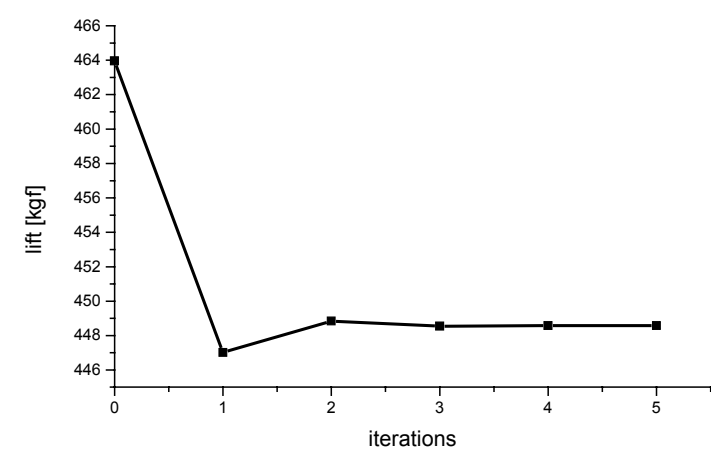

Fig.20 Lift value vs. number of iterations at $300 \mathrm{~km} / \mathrm{h}$

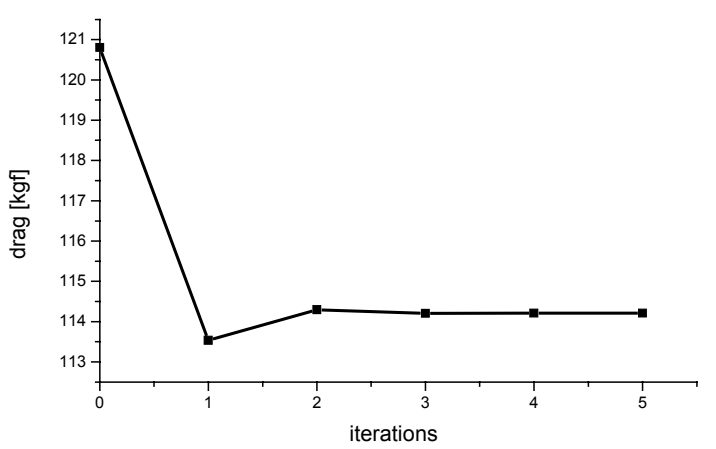

Fig 21: Drag value vs. number of iterations at $300 \mathrm{~km} / \mathrm{h}$

Then the percentage variation of the forces is displayed in fig. 22. As it can be observed, only 3 iterations are strictly necessary to reach the convergence of the procedure; this circumstance encourages the use of the integrated analysis to study the wings fluid dynamics since the additional cost required for the steady appears not excessive.

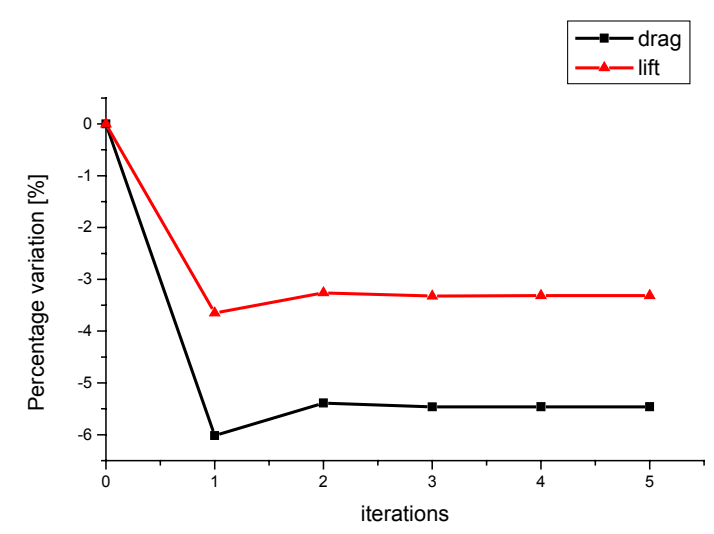

Fig 22: Percentage variation of drag and lift vs. number of iterations at $300 \mathrm{~km} / \mathrm{h}$

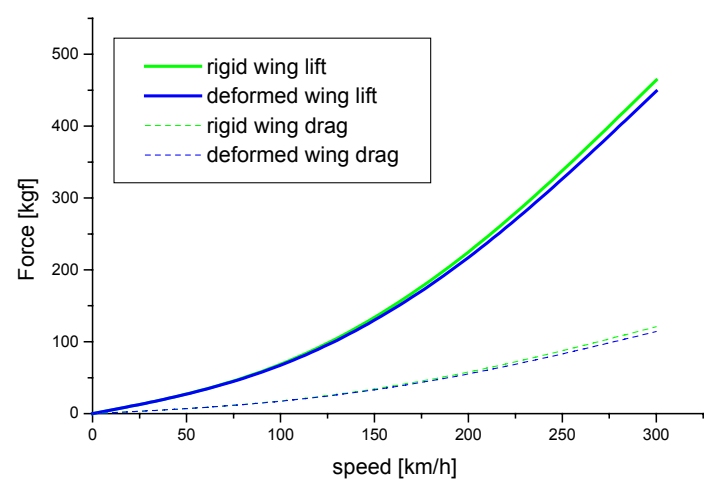

Fig 23: Lift and drag as function of the speed

In Fig. 23 the lift and drag trend vs. speed is reported: obviously the deviation from the rigid wing curves gets wider by raising the speed

As it can be observed, the predicted fluid dynamic forces offered by the wing change in a not negligible way by using an integrated approach: the deviation is obviously wider at higher speeds; it can be observed that at 300 $\mathrm{km} / \mathrm{h}$ drag reduces by $5.5 \%$, while lift reduces by $3.5 \%$ : the deformed wing then presents a higher efficiency than the rigid, even if it produces less desired effect, i.e. the lift. This circumstance would even justify the definition of an inverse numerical procedure which could give the undeformed wing geometry starting from the desired lift and deformed configuration.

For the sake of completeness, the analysis of the deformed wing in presence of installation errors was also performed. The results are synthesized in Table 2 and they are coherent with the previously described ones.

\begin{tabular}{|c|c|c|c|c|}
\hline & $\mathbf{1 0 0} \mathbf{~ k m} / \mathbf{h}$ & $\mathbf{2 0 0} \mathbf{~ k m} / \mathbf{h}$ & $\mathbf{3 0 0} \mathbf{~ k m} / \mathbf{h}$ & units \\
\hline lift + 2 & 58,38 & 220,8 & 512,2 & $\mathrm{kgf}$ \\
\hline lift + 1 & 54,47 & 205,9 & 480,88 & $\mathrm{kgf}$ \\
\hline lift & 50,77 & 200,89 & 448,58 & $\mathrm{kgf}$ \\
\hline lift - 1 & 45,87 & 177,2 & 412,92 & $\mathrm{kgf}$ \\
\hline lift - 2 & 42,22 & 160,98 & 375,82 & $\mathrm{kgf}$ \\
\hline drag+ 2 & 15 & 57,55 & 130,65 & $\mathrm{kgf}$ \\
\hline drag + 1 & 13,95 & 53,52 & 122,59 & $\mathrm{kgf}$ \\
\hline drag & 13 & 51,1 & 114,2 & $\mathrm{kgf}$ \\
\hline drag - 1 & 12,12 & 47,15 & 108,12 & $\mathrm{kgf}$ \\
\hline drag - 2 & 11,44 & 44,19 & 101,13 & $\mathrm{kgf}$ \\
\hline
\end{tabular}

Table 2: Drag and lift of the wing in presence of installation error (Notice that lift +1 means lift with an installation error of $+1^{\circ}$ ) 


\section{CONCLUSIONS}

A numerical fluid dynamic and structural coupled approach to study wings (or in general, objects) capable of being deformed has been presented in this paper.

The developed procedure allows the simulation of the fluid dynamic flow field around the wing by means of the Fluent CFD software, accounting for the wing deformation under the action of the fluid dynamic pressure loads evaluated by means of the MSCNASTRAN finite element method structural code. The final result consists of the actual wing geometry and a more realistic flow field around the object

The presented integrated methodology results mainly indicate that:

- The use of the integrated methodology is particularly suggested for high velocity vehicles (i.e. $300 \mathrm{~km} / \mathrm{h}$ ), while little difference in the fluid dynamic fields is observed at ordinary speeds (i.e. $100 \mathrm{~km} / \mathrm{h}$ )

- At high speeds lift and drag parameters are sensibly influenced by the wing deformation, since differences in the range of $4 \%-5 \%$ have been observed by applying the integrated methodology with respect to fluid dynamic pressure loads.

- The low number of iterations required to achieve the steady fluid dynamic and deformation fields make the use of the integrated procedure cost-effective especially in terms of time, as this procedure allows the reduction of the number of possible configurations to be tested in the wind-tunnels, which remain the decisive test to be get through.

Results obtained are encouraging and suggest to further investigate the possibilities offered by this procedure, as for example, the definition of an inverse procedure which could provide the undeformed geometry starting from the deformed one and the given constraints in terms of lift required.

\section{ACKNOWLEDGMENTS}

Authors wish to acknowledge Ing. Giulio De Simone for his helpful collaboration.

\section{REFERENCES}

1. B. Agathangelou, M. Gascoyne, "Aerodynamic Design Considerations of a Formula 1 Racing Car", SAE 980399

2. A. A. Boretti, G. Cantore, E. Matterelli, F. Preziosi, "Experimental and Computational Analysis of High Performance Motorcycle Engine”, SAE 962529.

3. D. Cambiaghi and M. Gadola, "Computer Aided Racing Car Design and Development at University of Brescia, Italy", SAE 942507
4. J. Corney, T. Lim, 3D Modeling with ACIS, SaxeCoburg Pubblications, 2001

5. G. De Simone, "Analisi Integrata dell'Interazione Fluido-Struttura di un Alettone per Autovetture ad Elevate Prestazioni" (in Italian). First level degree in Mechanical Engineering, University of Rome "Tor Vergata", a.y. 2002-2003

6. R.G. Dominy, "Aerodynamics of Grand Prix Cars", Proc.Instn.Mech.Engrs.1984 198D 1-7

7. J. Emery, J. Henrig, J.R. Erwin, R. Felix, "Systematic Two-Dimensional Cascade Tests of NACA 65Series Compressor Blades at Low Speeds", NACA REPORT 1368

8. Hucho Wolf-Heinrich, "Aerodynamics of Road Vehickes”, 1993 Fluid Mechanics Annual Review 25: 485-537

9. W. F. Milliken and D. L. Milliken, "Race Car Vehicle Dynaminc", SAE 1994.

10. V. Mulone "Metodo Cinetico Turbolento BGK a Reticolo Localmente Infittito per l'Analisi dell'Efflusso in Turbomacchine Assiali" (In Italian). Degree Thesis in Mechanical Engineering, University of Rome "La Sapienza", a.y. 1999-2000

11. G. Pahl, W. Beitz, Engineering Design, a Systematic Approach, 1999, Springer ed.

12. M. Ramnefors et al., "Accuracy of Drag Predictions on Cars Using CFD - Effect of Grid Refinement and Turbulence Models", SAE Paper 960681

13. C.T. Shaw, "Predicting Vehicle Aerodynamics Using Computational Fluid Dynamics - A User's perspective." Automotive Aerodynamics 1988 Society of Automotive Engineers

14. F. Werner, S. Frik, J. Schulze, "Aerodynamic Optimization of the Opel Calibra ITC Racing Car Using Experiments and Computational Fluid Dynamics", SAE 980040

15. M. D. Woodbridge, R. B. Miller, "The Aerodynamic Optimization of a Successful Imsa GT Race Car", SAE 962518.

16. P.A.Newman, G.J.W.Hou, A.C.Taylor, "Observations Regarding Use of Advanced CFD Analysis and Design Codes in MDO", ICASE Report 96-16, NASA CR 198293

17. C.R.Gumbert,G.J.W.Hou,P.A.Newman,

"Simultaneous Aerodynamic Analysis and Design Optimization (SAADO) for a 3-D Flexible Wing", $39^{\text {th }}$ Aerospace Science Meeting \& Exhibit, Paper $n$. AIAA 2001-1107, 2001

18. J.B.Barlow, W.H.Rae Jr., A.Pope, "Low-Speed Wind Tunnel Testing", Wiley \& Sons ISBN 0-471-5574-9

19. MSC/NASTRAN Version 70.5 Quick Reference Guide, The MacNeal-Schwendler Corporation, 1998 20. FLUENT Inc. "GAMBIT and FLUENT User's guide" 